tion time was 62.2 (45-75) minutes. The flap design differed from a conventional design (figure $1 A$ ) in that parallel lines were vertically drawn from the lateral borders with a length that was 1.0-1.5-fold longer than the vertical diameter of defects (figure $1 B$ ), and the Burow's triangles were excised depending on tissue redundancy.

Relative to conventional design, our method is superior with respect to the traction vector applied on the cheek (figure $1 A, B$ ). In the field of aesthetic surgery, recent refinements in rhytidectomy have yielded a simple but effective technique termed "the minimal access cranial suspension lift" [6]. This procedure takes advantage of the vertical vector, as with our design, and avoids horizontal traction, whereas traditional rhytidectomy comprises horizontal traction, similar to the conventional design and results in redraping in an oblique direction [6]. Moreover, due to greater flap extensibility, a relatively smaller area of undermining was necessary compared with the conventional method, which could prevent postoperative bleeding complications. Although there may be controversy over vertically lined parallel scars, our procedure follows the guiding principles that the surgical margins are hidden within the natural borders of the cheek unit, and the vertical lines follow relaxed skin tension lines [7]. In fact, skin laxity minimized postoperative scar formation, resulting in superior cosmetic acceptability, so long as it was performed in elderly patients (figure 1C-E).

Since NMSCs are rarely fatal, there is an ongoing debate as to whether short-life expectancy should be taken into account in treatment decision-making [5]. However, the fact that the periauricular area is an independent highrisk factor for SCC should be considered [2]. Therefore, the invasiveness of procedures should not be determined based on patients' age and a one-size-fits-all basis [5], and complete disease control should always be sought [2]. Indeed, we experienced two cases of local recurrence or lymph node metastasis after surgery; the former (Case 4) (figure $1 E$ ) was managed by extended resection followed by chemoradiotherapy using fluorouracil plus cisplatin for up to 30 months (figure $1 E$ ), and the latter remains in complete remission, 10 months after cervical lymph node dissection (Case 7). Therefore, following meticulous consideration and close observation, our simple and efficient method could be an attractive reconstructive option.

Disclosure. Financial support: none. Conflicts of interest: none.

Department of Dermatology,
Faculty of Medicine, University of
Tsukuba, Tennodai, Tsukuba,
Ibaraki 305-8575, Japan
<yosuke.ishitsuka@md.tsukuba.ac.jp>

Yasuko NIIJIMA Yosuke ISHITSUKA Sae INOUE Hiroshi MARUYAMA Yoshiyuki NAKAMURA Yasuhiro FUJISAWA Manabu FUJIMOTO

1. Rogers HW, Weinstock MA, Feldman SR, Coldiron BM. Incidence estimate of nonmelanoma skin cancer (keratinocyte carcinomas) in the U.S. population, 2012. JAMA Dermatol 2015; 151: 1081-6.

2. Work G, Invited R, Kim JYS, et al. Guidelines of care for the management of cutaneous squamous cell carcinoma. J Am Acad Dermatol 2018; 78: 560-78.
3. Inouye SK, Westendorp RG, Saczynski JS. Delirium in elderly people. Lancet 2014;383:911-22.

4. Imadojemu S, Sarwer DB, Percec I, et al. Influence of surgical and minimally invasive facial cosmetic procedures on psychosocial outcomes: a systematic review. JAMA Dermatol 2013; 149: 1325-33.

5. Linos E, Chren MM, Stijacic Cenzer I, Covinsky KE. Skin cancer in U.S. elderly adults: does life expectancy play a role in treatment decisions? J Am Geriatr Soc 2016 ; 64 : 1610-5.

6. Verpaele A, Tonnard P, Gaia S, Guerao FP, Pirayesh A. The third suture in MACS-lifting: making midface-lifting simple and safe. J Plast Reconstr Aesthet Surg 2007; 60: 1287-95.

7. Robinson JK, Hanke CW, Siegel DM, Fratila A, Bhatia AC, Rohrer TE. Surgery of the Skin E-Book: Procedural Dermatology: Elsevier Health Sciences, 2014.

doi:10.1684/ejd.2019.3659

\section{Successful treatment of pustulotic arthro-osteitis with apremilast: a case report with follow-up MRI}

Pustulotic arthro-osteitis (PAO) is an inflammatory arthroosteopathy, which is seen in patients with palmoplantar pustular psoriasis (PPP). Here, we report a case of bone marrow oedema due to PAO, which was successfully treated with apremilast.

A 65-year-old woman visited our department with palmoplantar exanthema and joint pain. During her childhood, she underwent tonsillectomy for tonsillar hypertrophy. Twentyfive years ago, pustules, erythema, and scales appeared on her palms and soles, and her symptoms partially responded to a combination treatment of topical corticosteroid and topical vitamin $\mathrm{D}_{3}$. However, the symptoms persisted, and she consulted our department for pain and itching on the palms and soles.

Physical examination revealed pustules, erythema, and scales on the palms and soles (figure 1A). Nail pitting, splinter haemorrhage, Beau's lines, and distal leukonychia were also seen on the left middle and little fingers (figure $1 F$ ). Her serum was negative for anti-cyclic citrullinated peptide antibody on enzyme-linked immunosorbent assay (ELISA) and rheumatoid factor on latex coagulating nephelometry. Biopsy specimen obtained from the palm showed an intraepidermal pustule.

Initially, the patient was treated with a combination of topical steroid, topical vitamin $\mathrm{D}_{3}$, and excimer light. Excimer light irradiation was administered 20 times (total: $10.85 \mathrm{~J} / \mathrm{cm}^{2}$ ). However, the symptoms persisted (figure $1 B$ ). Seven months after her first visit to our department, arthralgia appeared on her right knee, anterior chest, and both feet. Magnetic resonance imaging (MRI) revealed bone marrow oedema on the proximal femoral head of the right tibia (figure $1 D$ ).

The patient was diagnosed with PAO and nail psoriasis accompanying PPP and was treated with oral apremilast. The rash on the palms and soles almost cleared within four weeks of apremilast administration (figure 1C). Arthralgia on the right knee, bilateral transverse tarsal joints, and sternoclavicular joints was relieved within a month. The 

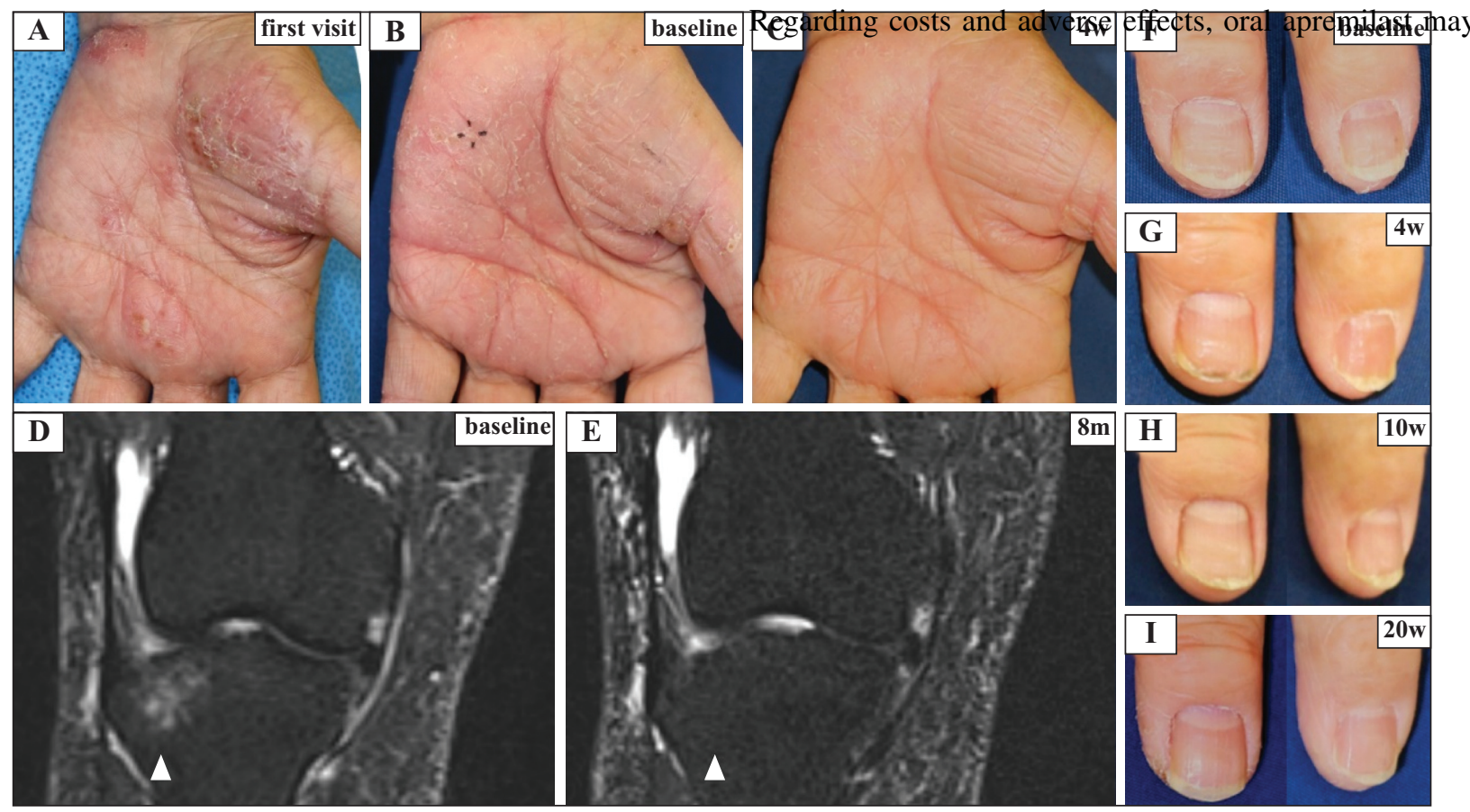

Figure 1. Chronological changes of exanthema and MRI findings. A-C) Clinical photographs of the patient's left palm; at her first visit to our hospital, erythematous plaques with scales and pustules were seen (A); seven months after her first visit to our hospital, oral apremilast was initiated, and topical steroid, vitamin $\mathrm{D}_{3}$, and excimer light irradiation $\left(20\right.$ times; total: $\left.10.85 \mathrm{~J} / \mathrm{cm}^{2}\right)$ improved the pustules, however, the scaly erythema persisted (B); four weeks after initiation of oral apremilast, exanthema was almost cleared (C). D, E) MRI of the patient's right knee; seven months after her first visit to our hospital, oral apremilast was initiated, and bone marrow oedema was seen in the proximal femoral head of the right tibia (D) (white arrowhead); eight months after initiation of oral apremilast, bone marrow oedema disappeared (E). F-I) Clinical images of the nails of the patient's left middle finger (left column) and little finger (right column); seven months after her first visit to our hospital, oral apremilast was initiated, and nail pitting, splinter haemorrhage, Beau's lines, and distal leukonychia were seen (F); oral apremilast gradually improved the nail symptoms, and the findings on the fingernails almost disappeared within 20 weeks (G-I).

symptoms on her fingernails almost disappeared within 20 weeks (figure $1 G-I$ ). Follow-up MRI at eight months after the initiation of oral apremilast showed disappearance of bone marrow oedema on the proximal femoral head of the right tibia (figure $1 E$ ).

PPP is also known as palmoplantar pustulosis, and PPP and PAO are closely related to psoriasis and psoriatic arthritis [1]. However, whether PPP is a variant of psoriasis or a distinct condition remains controversial [2]. Severe cases of PPP are treated with oral retinoid, cyclosporine, methotrexate, and colchicine [3]. In 2019, Eto et al. reported three cases of PPP successfully treated with apremilast, which is a PDE-4 inhibitor broadly used for the treatment of psoriasis [4]. Similar to the previous report, in the present case, apremilast was found to improve palmoplantar exanthema and nail psoriasis.

PAO is associated with $10-30 \%$ of PPP cases in the Japanese population [5]. Adamo et al. [6] reported a case of PAO treated with apremilast in 2018, and mentioned that suppression of proinflammatory cytokines by apremilast may lead to pan-cytokine and multi-cell immunomodulation. However, no report describes the detailed radiological features of arthritis in PAO patients treated with apremilast.

To our knowledge, this is the second case report of PAO treated with apremilast and is the first to describe the radiologically-proven improvement of PAO by apremilast.

be superior to biologics. Therefore, oral apremilast can be a useful therapeutic option for refractory arthro-osteitis, nail deformity, and palmoplantar exanthema in patients with $\mathrm{PPP}$ and PAO.

Acknowledgements and disclosures. Funding sources: none. Conflicts of interest: none. Acknowledgements: none

${ }^{1}$ Department of Dermatology, Aichi
Medical University, Aichi, Japan
${ }^{2}$ Department of Dermatology,
Nagoya University, Aichi, Japan
<yh_jp_hirot@yahoo.co.jp>

1. Sonozaki H, Kawashima M, Hongo O, et al. Incidence of arthroosteitis in patients with pustulosis palmaris et plantaris. Ann Rheum Dis 1981; 40: 554-7.

2. Griffiths CE, Christophers E, Barker JN, et al. A classification of psoriasis vulgaris according to phenotype. $\mathrm{Br} J$ Dermatol 2007; 156: 258-62.

3. Engin B, Așkın Ö, Tüzün Y. Palmoplantar psoriasis. Clin Dermatol 2017; 35: 19-27.

4. Eto $A$, Nakao M, Furve M. Three cases of palmoplantar pustulosis successfully treated with apremilast. J Dermatol 2019; 46: e29-30. 
5. Ohashi T, Hiraiwa T, Yamamoto T. Low prevalence of anti-cyclic citrullinated peptide antibodies in Japanese patients with pustulotic arthro-osteitis. Indian J Dermatol 2016;61:221-2.

6. Adamo S, Nilsson J, Krebs A, et al. Successful treatment of SAPHO syndrome with apremilast. Br J Dermatol 2018; 179:959-62.

doi:10.1684/ejd.2019.3660

\section{Treatment of atopic dermatitis with dupilumab in Taiwan: dynamic changes of IgE levels as a potential response biomarker}

Atopic dermatitis (AD) is an inflammatory skin condition affecting a significant percentage of the population [1]. Dupilumab is a targeted biologic agent approved by the FDA and EMA for the treatment of moderate-to-severe AD. Dupilumab targets IL-4 receptor $\alpha$ and inhibits the IL-4 and IL-13-related cascades that are critically involved in the pathogenesis of $\mathrm{AD}$, including inflammation, pruritus, and IgE production. Although dupilumab demonstrated efficacy in different clinical studies, a biomarker that correlates with its therapeutic response is currently unavailable.
In this study, we enrolled seven adult Taiwanese patients with AD for three months with dupilumab treatment. Our results confirm that dupilumab has a good therapeutic and safety profile for treating $\mathrm{AD}$, and suggest that dynamic changes of total IgE levels may serve as a biomarker reflecting therapeutic response to dupilumab treatment.

Patients older than 20 years of age with inadequately controlled moderate-to-severe $\mathrm{AD}$ for more than three years were recruited (KMUHIRB-F(I)-20180044). Patients received a dupilumab 600-mg loading dose and subsequent 300-mg dupilumab dose every two weeks for 12 weeks. Scores for Eczema Area and Severity Index (EASI) and Dermatology Life Quality Index (DLQI), as well as pruritus assessed by numerical rating scale (NRS) were documented at baseline and every two weeks during the study. Laboratory data, including haemogram, biochemistry, and IgE, were evaluated. Systemic antihistamine and topical steroid use were allowed if they were part of the regular regimen at baseline. No systemic immunosuppressant including corticosteroid was allowed.

Overall, 6/7 achieved EASI-75 after 12 weeks of treatment (figure 1). This efficacy is better than previous reports $[2,3]$. Allowing baseline regimen use likely contributed to this higher efficacy. Besides Patient 1 (who stopped taking antihistamines six weeks after dupilumab treatment initiation), no other patients modified their medication during the study. A disparity between EASI and NRS scores before the initiation of treatment was noted.

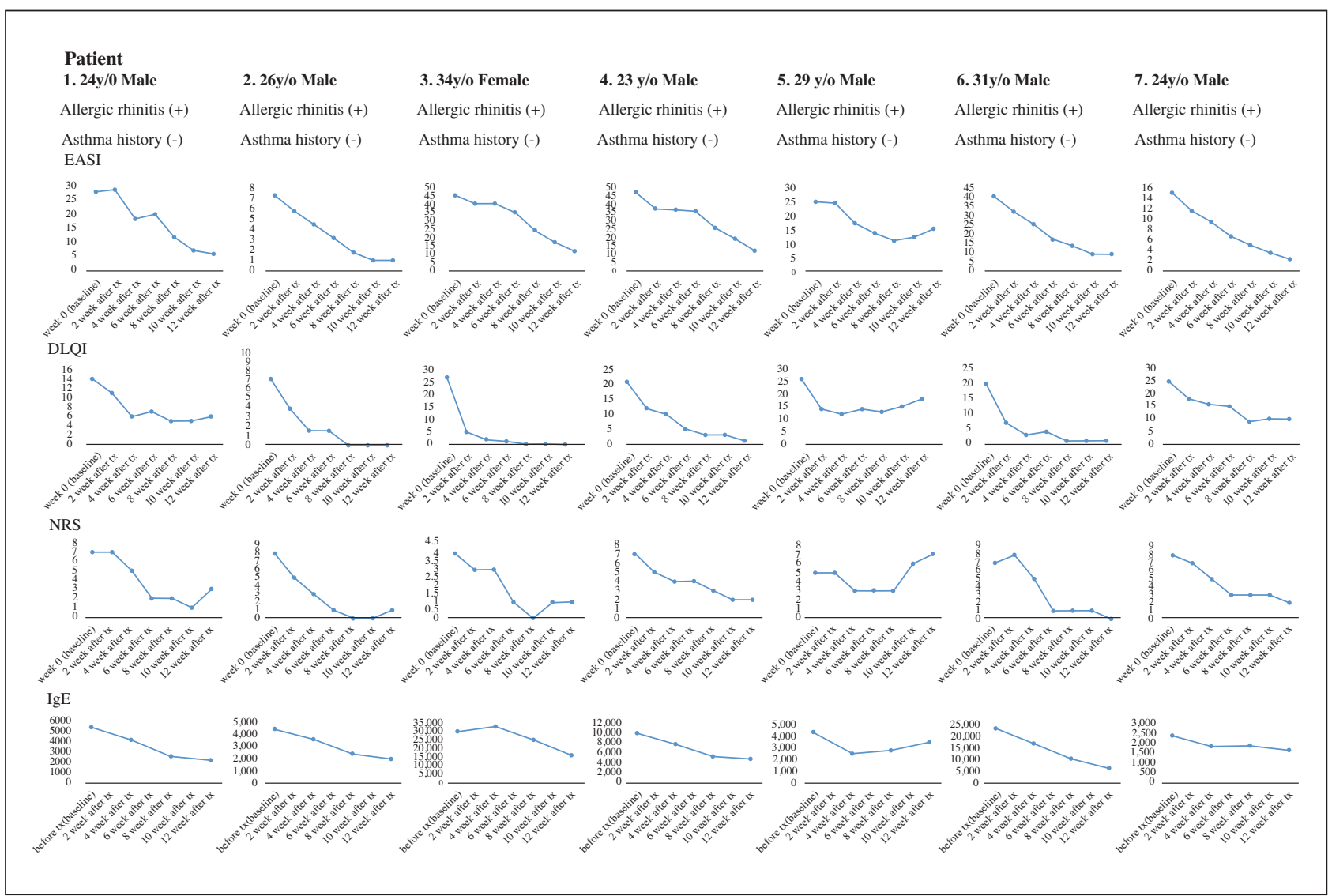

Figure 1. The clinical and laboratory profiles of the patients in this study. Each column represents the profiles of each individual patient. 